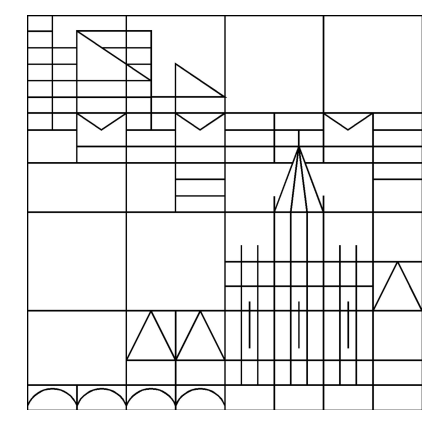

Further applications of the Cauchon algorithm to rank determination and bidiagonal factorization

\author{
Mohammad Adm \\ Khawla Al Muhtaseb \\ Ayed Abedel Ghani \\ Shaun Fallat \\ Jürgen Garloff
}

Konstanzer Schriften in Mathematik

Nr. 373, Februar 2018

ISSN 1430-3558 



\title{
Further applications of the Cauchon algorithm to rank determination and bidiagonal factorization
}

\author{
Mohammad Adma a,b, Khawla Al Muhtaseb ${ }^{\mathrm{d}}$, Ayed Abedel Ghani ${ }^{\mathrm{a}}$, Shaun \\ Fallat $^{\mathrm{d}}$, Jürgen Garloff , $^{\mathrm{b}}$ \\ ${ }^{a}$ Department of Applied Mathematics and Physics, Palestine Polytechnic University, \\ Hebron, Palestine \\ ${ }^{b}$ Department of Mathematics and Statistics, University of Konstanz, D-78464 Konstanz, \\ Germany \\ ${ }^{c}$ Institute of Applied Research, University of Applied Sciences / HTWG Konstanz, \\ D-78405 Konstanz, Germany \\ ${ }^{d}$ Department of Mathematics and Statistics, University of Regina, Regina, SK, S4S 0A2 \\ Canada
}

\begin{abstract}
For a class of matrices connected with Cauchon diagrams, Cauchon matrices, and the Cauchon algorithm, a method for determining the rank, and for checking a set of consecutive row (or column) vectors for linear independence is presented. Cauchon diagrams are also linked to the elementary bidiagonal factorization of a matrix and to certain types of rank conditions associated with submatrices called descending rank conditions.
\end{abstract}

Keywords:

Rank, Cauchon matrix, Cauchon diagram, Cauchon algorithm, linear independence, elementary bidiagonal factorization

2010 MSC: 15A03, 15A23, 15B99

*Corresponding author: Mohammad Adm

Email addresses: mjamathe@yahoo.com (Mohammad Adm), khawla@ppu.edu (Khawla Al Muhtaseb), ayed42@ppu.edu (Ayed Abedel Ghani), shaun.fallat@uregina.ca (Shaun Fallat), juergen.garloff@htwg-konstanz.de (Jürgen Garloff) 


\section{Introduction}

A fundamental problem in mathematics and its applications is the determination of the rank of a matrix. The rank of a given real $n$-by- $m$ matrix can be determined by, for example, Gaussian elimination. In this paper, we present an alternative approach to calculating the rank and bidiagonal factorization for matrices that admit a Cauchon matrix upon application of the Cauchon algorithm. This method relies on Cauchon diagrams, Cauchon matrices, and the so-called (condensed) Cauchon algorithm. Like Gaussian elimination, single computations of the Cauchon algorithm can be represented as the computation of minors of order two. In this way, a series of intermediate matrices is produced. The algorithm culminates with a matrix from which we can easily determine the rank of the original matrix.

This algorithm was applied in [1]-[4], [9], [11], [13] to problems related to totally nonnegative matrices, that is, matrices having all their minors nonnegative. For properties of these matrices, the reader is referred to the monographs [7] or [12]. In [1], [3], a condensed form of the Cauchon algorithm was derived by which the amount of required arithmetic operations was reduced by one order of magnitude to bring it in line with the complexity of performing conventional Gaussian elimination, viz. $O\left(n^{3}\right)$ for a nonsingular $n$-by- $n$ matrix. In the first part of our paper, we apply the condensed form of the Cauchon algorithm to matrices that admit a Cauchon matrix upon application of this algorithm in order to determine their ranks and linear independence of sets of consecutive row vectors. The class of matrices that admit a Cauchon matrix upon application of the Cauchon algorithm contains the totally nonnegative matrices [11], totally nonnpositive matrices (matrices with all of its minors nonpositive) [4], and matrices satisfying the descending rank conditions, see Section 4.

In the second part of the paper, we link Cauchon diagrams to the factorization of matrices into elementary bidiagonal matrices, namely, one-banded, unit diagonal matrices having at most one nonzero off-diagonal entry, see [7, Chapter 2], [12, Chapter 6]. Elementary bidiagonal factorization itself is linked to certain types of rank conditions associated with submatrices called descending rank conditions [10]; see [8] for an earlier paper discussing these conditions. We directly link Cauchon diagrams (or matrices) to the descending rank conditions hereby reducing the complexity by some orders of magnitude.

We point out that all of our results are valid if $\mathbb{R}$ is replaced by any field 
of characteristic zero.

The organization of our paper is as follows: In the next section we introduce the notation used in our paper and provide some auxiliary results and the concept of a lacunary sequence which are employed in the subsequent sections. In Section 3 we use lacunary sequences in order to determine the rank of a certain matrix, and to check the linear independence of its row or column vectors. Finally, in Section 4, we present relations between Cauchon diagrams, elementary bidiagonal factorization, and the descending rank conditions.

\section{Notation and Auxiliary Results}

\subsection{Notation}

We now introduce the notation used in our paper. For integers $\kappa, n$, we denote by $Q_{\kappa, n}$ the set of all strictly increasing sequences of $\kappa$ integers chosen from $\{1,2, \ldots, n\}$. We employ the set theoretic notation to denote less precisely but intuitively sequences from $Q_{\kappa, n}$, the membership $\epsilon$ in a sequence, and the union $\cup$, intersection $\cap$, and difference $\backslash$ of two sequences, where we consider the resulting sequences as strictly increasing ordered. For $\alpha \in Q_{\kappa, n}$, we put $\alpha^{c}=\{1, \ldots, n\} \backslash \alpha$. For a sequence $\alpha,|\alpha|$ denotes the number of its members. Let $A$ be a real $n$-by- $m$ matrix. For $\alpha=\left\{\alpha_{1}, \alpha_{2}, \ldots, \alpha_{\kappa}\right\}, \beta=\left\{\beta_{1}, \beta_{2}, \ldots, \beta_{\kappa}\right\} \in Q_{\kappa, n}$, we denote by $A[\alpha \mid \beta]$ the $\kappa$-by- $\kappa$ submatrix of $A$ contained in the rows indexed by $\alpha_{1}, \alpha_{2}, \ldots, \alpha_{\kappa}$ and columns indexed by $\beta_{1}, \beta_{2}, \ldots, \beta_{\kappa}$. We suppress the brackets when we enumerate the indices explicitly. We set $A^{\#}:=T A T$, where $T=\left(t_{i j}\right)$ is the permutation matrix of order $n$ (antidiagonal matrix) with $t_{i j}:=\delta_{i, n-j+1}$, $i, j=1, \ldots, n$. By $E_{i j}$ we denote the elementary bidiagonal $(E B)$ matrix in $\mathbb{R}^{n, m}$ which has in position $(i, j)$ a one, while all other entries are zero. A matrix $A \in \mathbb{R}^{n, m}$ is called totally nonnegative if $\operatorname{det} A[\alpha \mid \beta] \geq 0$, for all $\alpha \in Q_{\kappa, n}, \beta \in Q_{\kappa, m}, \kappa=1,2, \ldots, n^{\prime}$, where $n^{\prime}:=\min \{n, m\}$. We denote by $\leq$ the lexicographic order on $\mathbb{N}^{2}$, i.e.,

$$
(g, h) \leq(i, j): \Leftrightarrow(g<i) \text { or }(g=i \text { and } h \leq j) .
$$

\subsection{Auxiliary Results}

In this subsection we compile a list of relevant facts and existing related results that will be employed in Sections 3 and 4 which contain the main contributions in this work. 
Lemma 2.1. [5, Corollary 1] Let $A$ be an n-by-m matrix such that $\operatorname{det} A[\gamma \mid \delta] \neq$ 0 , where $\gamma \in Q_{\kappa, n}$ and $\delta \in Q_{\kappa, m}$ and let $B=\left(b_{i j}\right)$ be the matrix obtained from $A$ by setting

$$
b_{i j}:=\frac{\operatorname{det} A[\gamma \cup\{i\} \mid \delta \cup\{j\}]}{\operatorname{det} A[\gamma \mid \delta]}, \text { for all }(i, j) \in \gamma^{c} \times \delta^{c} .
$$

Then it holds that

$$
\operatorname{rank} A\left[\gamma \cup \gamma^{\prime} \mid \delta \cup \delta^{\prime}\right]=|\gamma|+\operatorname{rank} B\left[\gamma^{\prime} \mid \delta^{\prime}\right]
$$

where $\gamma^{\prime}$ and $\delta^{\prime}$ are strictly increasing sequences taken from $\{1, \ldots, n\}$ and $\{1, \ldots, m\}$, respectively, such that $\gamma \cap \gamma^{\prime}=\delta \cap \delta^{\prime}=\phi$.

We now turn our attention to Cauchon diagrams and the (condensed) Cauchon algorithm which will play a key role moving forward. Cauchon diagrams are named after Gérard Cauchon who used these diagrams in his study of quantum matrices [6]. It should be noted that they appear also independently in the work of Alexander Postnikov [13] who called them Lediagrams.

Definition 2.2. An n-by-m Cauchon diagram $C$ is an n-by-m grid consisting of nm squares colored black and white, where each black square has the property that either every square to its left (in the same row) or every square above it (in the same column) is black.

We denote by $\mathcal{C}_{n, m}$ the set of all $n$-by- $m$ Cauchon diagrams. We fix positions in a Cauchon diagram in the following way: For $C \in \mathcal{C}_{n, m}$ and $i \in\{1, \ldots, n\}, j \in\{1, \ldots, m\},(i, j) \in C$ if the square in row $i$ and column $j$ is black. Here we use the usual matrix notation for the $(i, j)$ position in a Cauchon diagram, i.e., the square in the $(1,1)$ position of the Cauchon diagram is in its top left corner.

Definition 2.3. Let $A \in \mathbb{R}^{n, m}$ and let $C \in \mathcal{C}_{n, m}$. We say that $A$ is a Cauchon matrix associated with the Cauchon diagram $C$ if for all $(i, j), i \in\{1, \ldots, n\}$, $j \in\{1, \ldots, m\}$, we have $a_{i j}=0$ if and only if $(i, j) \in C$. If $A$ is a Cauchon matrix associated with an unspecified Cauchon diagram, we just say that $A$ is a Cauchon matrix. 
Algorithm 2.4. (Condensed form of the Cauchon Algorithm) [1, Algorithm 3.3], [3, Algorithm 3.2] Let $A=\left(a_{i j}\right) \in \mathbb{R}^{n, m}$. Set $A^{(n)}:=A$.

For $k=n-1, \ldots, 1$ define $A^{(k)}=\left(a_{i j}^{(k)}\right) \in \mathbb{R}^{n, m}$ as follows:

For $j=1, \ldots, m-1$,

set $s_{j}:=\min \left\{h \in\{j+1, \ldots, m\} \mid a_{k+1, h}^{(k+1)} \neq 0\right\}$ (we set $s_{j}:=\infty$ if this set is empty),

for $i=1, \ldots, k$,

$$
a_{i j}^{(k)}:= \begin{cases}a_{i j}^{(k+1)}-\frac{a_{k+1, j}^{(k+1)} a_{i s_{j}}^{(k+1)}}{a_{k+1, s_{j}}^{(k+1)}} & \text { if } s_{j}<\infty, \\ a_{i j}^{(k+1)} & \text { if } s_{j}=\infty,\end{cases}
$$

and for $i=k+1, \ldots, n, j=1, \ldots, m$, and $i=1, \ldots, k, j=m$

$$
a_{i j}^{(k)}:=a_{i j}^{(k+1)}
$$

Put $\tilde{A}:=A^{(1)} ; \tilde{A}$ is called the matrix obtained from $A$ (by the condensed form of the Cauchon Algorithm).

We recall from [11] the definition of a lacunary sequence associated with Cauchon diagrams.

Definition 2.5. Let $C \in \mathcal{C}_{n, m}$. We say that a sequence

$$
\gamma:=\left(\left(i_{k}, j_{k}\right), \quad k=0,1, \ldots, t\right)
$$

which is strictly increasing in both arguments is a lacunary sequence with respect to $C$ if the following conditions hold:

1. $\left(i_{k}, j_{k}\right) \notin C, k=1, \ldots, t$;

2. $(i, j) \in C$ for $i_{t}<i \leq n$ and $j_{t}<j \leq m$.

3. Let $s \in\{1, \ldots, t-1\}$. Then $(i, j) \in C$

(a) either for all $(i, j), i_{s}<i<i_{s+1}$ and $j_{s}<j$, or for all $(i, j), i_{s}<i<i_{s+1}$ and $j_{0} \leq j<j_{s+1}$

and

(b) either for all $(i, j), i_{s}<i$ and $j_{s}<j<j_{s+1}$

or for all $(i, j), i<i_{s+1}$, and $j_{s}<j<j_{s+1}$. 
We call $t$ the length of $\gamma$.

In [3], [11] algorithms are presented which construct for a given Cauchon diagram $C$ and any fixed square of $C$ a lacunary sequence (with respect to $C$ ) starting at this square. The following proposition was given in [11, Proposition 4.1] with a sign condition which was removed in [4, Proposition 4.11].

Proposition 2.6. Let $A \in \mathbb{R}^{n, m}$ and $C \in \mathcal{C}_{n, m}$. For each position in $C$ fix a lacunary sequence $\gamma=\left(\left(i_{0}, j_{0}\right),\left(i_{1}, j_{1}\right), \ldots,\left(i_{t}, j_{t}\right)\right)$ with respect to $C$ starting at this position. Assume that for all $\left(i_{0}, j_{0}\right)$, we have

$$
0=\operatorname{det} A\left[i_{0}, i_{1}, \ldots, i_{t} \mid j_{0}, j_{1}, \ldots, j_{t}\right] \quad \text { if and only if }\left(i_{0}, j_{0}\right) \in C .
$$

Then

$$
\operatorname{det} A\left[i_{0}, i_{1}, \ldots, i_{t} \mid j_{0}, j_{1}, \ldots, j_{t}\right]=\tilde{a}_{i_{0}, j_{0}} \cdot \tilde{a}_{i_{1}, j_{1}} \cdots \tilde{a}_{i_{t}, j_{t}}
$$

holds for all lacunary sequences $\gamma$ given by (2).

\section{Rank Determination}

We begin this section with a construction of a uniquely determined sequence related to the rank of certain matrices. All of the matrices $A$ that are considered in this and the next section are assumed to be different from the null matrix. We point out that all of our results are valid if $\mathbb{R}$ is replaced by any field of characteristic zero.

Procedure 3.1. Let $A \in \mathbb{R}^{n, m}$ be a Cauchon matrix. Construct the sequence

$$
\gamma=\left(\left(i_{p}, j_{p}\right), \ldots,\left(i_{0}, j_{0}\right)\right)
$$

as follows:

- $\operatorname{Put}\left(i_{-1}, j_{-1}\right):=(n+1, m+1)$.

- For $k=0,1, \ldots, p$, define

$$
M_{k}:=\left\{(i, j) \mid 1 \leq i<i_{k-1}, \quad 1 \leq j<j_{k-1}, a_{i j} \neq 0\right\} .
$$

If $M_{k}=\phi$, put $p:=k-1$. Otherwise, put $\left(i_{k}, j_{k}\right):=\max M_{k}$, where the maximum is taken with respect to the lexicographical order. 
Lemma 3.2. Let $A \in \mathbb{R}^{n, m}$ be a Cauchon matrix and $\gamma$ given by (4) be the sequence obtained by applying Procedure 3.1. Then $\gamma$ is a lacunary sequence with respect to $C_{A}$.

Proof. Conditions 1 and 2 of Definition 2.5 hold trivially. For Condition 3 we consider only the part of the sequence between any two adjacent pairs $\left(i_{s+1}, j_{s+1}\right)$ and $\left(i_{s}, j_{s}\right)$ for $s=p-1, \ldots, 0$. We distinguish the following three cases:

Case 1. $i_{s}>i_{s+1}+1$ and $j_{s}=j_{s+1}+1$. In this case $a_{i, t_{2}}=0$ for all $i_{s+1}<i<i_{s}$ and $t_{2}<j_{s}$.

Case 2. $i_{s}=i_{s+1}+1$ and $j_{s}>j_{s+1}+1$. In this case $a_{t_{1}, j}=0$ for all $t_{1}<i_{s}$, and $j_{s+1}<j<j_{s}$.

Case 3. $i_{s}>i_{s+1}+1$ and $j_{s}>j_{s+1}+1$. In this case $a_{i, t_{2}}=0$ for all $i_{s+1}<i<i_{s}$ and $t_{2}<j_{s}$, and $a_{t_{1}, j}=0$ for all $t_{1}<i_{s}$ and $j_{s+1}<j<j_{s}$.

Hence in all three cases Condition 3 of Definition 2.5 holds. Therefore, $\gamma$ is a lacunary sequence.

Corollary 3.3. Let $A \in \mathbb{R}^{n, m}$ be such that $\tilde{A}$ is a Cauchon matrix and let $\gamma=\left(\left(i_{k}, j_{k}\right), \quad k=0,1, \ldots, t\right)$ be a lacunary sequence. Then the following representation holds:

$$
\operatorname{det} A\left[i_{0}, \ldots, i_{t} \mid j_{0}, \ldots, j_{t}\right]=\tilde{a}_{i_{0}, j_{0}} \tilde{a}_{i_{1}, j_{1}} \cdots \tilde{a}_{i_{t}, j_{t}} .
$$

Proof. For each position in $C_{\tilde{A}}$ we fix a lacunary sequence (with respect to $\left.C_{\tilde{A}}\right)$ starting at this position. By Proposition 2.6, we only have to show that for all $\left(i_{0}, j_{0}\right)$

$$
0=\operatorname{det} A\left[i_{0}, i_{1}, \ldots, i_{t} \mid j_{0}, j_{1}, \ldots, j_{t}\right] \text { if and only if }\left(i_{0}, j_{0}\right) \in C_{\tilde{A}} .
$$

Assume to the contrary and let $(i, j)$ be the maximum position in $C_{\tilde{A}}$ with respect to the lexicographical order for which the assumption is not fulfilled, i.e., $\tilde{a}_{i j} \neq 0$ but $\operatorname{det} A\left[i_{0}, \ldots, i_{t} \mid j_{0}, \ldots, j_{t}\right]=0$ with $\left(i_{0}, j_{0}\right)=(i, j)$ and $i_{0}<n$ and $j_{0}<m$ (the case $\tilde{a}_{i j}=0$ and $\operatorname{det} A\left[i_{0}, \ldots, i_{t} \mid j_{0}, \ldots, j_{t}\right] \neq 0$ follows analogously). Then $B:=A[i, \ldots, n \mid j, \ldots, m]+x E_{11}$ for some $x \neq 0$ fulfills the hypothesis of Proposition 2.6 and we may conclude that

$$
\operatorname{det} B\left[i_{0}, \ldots, i_{t} \mid j_{0}, \ldots, j_{t}\right]=\left(\tilde{a}_{i_{0}, j_{0}}+x\right) \cdot \tilde{a}_{i_{1}, j_{1}} \cdots \tilde{a}_{i_{t}, j_{t}} .
$$

On the other hand, Laplacian expansion gives

$\operatorname{det} B\left[i_{0}, \ldots, i_{t} \mid j_{0}, \ldots, j_{t}\right]=\operatorname{det} A\left[i_{0}, \ldots, i_{t} \mid j_{0}, \ldots, j_{t}\right]+x \operatorname{det} A\left[i_{1}, \ldots, i_{t} \mid j_{1}, \ldots, j_{t}\right]$. 
By our hypothesis, the assumption of Proposition 2.6 is fulfilled for the lacunary sequence $\left(\left(i_{1}, j_{1}\right), \ldots,\left(i_{t}, j_{t}\right)\right)$ and we may conclude by $(5)$ that

$$
\operatorname{det} A\left[i_{0}, \ldots, i_{t} \mid j_{0}, \ldots, j_{t}\right]=\tilde{a}_{i_{0}, j_{0}} \cdot \tilde{a}_{i_{1}, j_{1}} \cdots \tilde{a}_{i_{t}, j_{t}} \cdot
$$

Since by our assumption the left-hand side is zero, while the right-hand side is not equal zero we reach a contradiction.

Theorem 3.4. Let $A \in \mathbb{R}^{n, m}$ be such that $\tilde{A}$ is a Cauchon matrix. Then $\operatorname{rank} A=p+1$, where $p$ is the length of the sequence which is obtained by application of Procedure 3.1 to $\tilde{A}$.

Proof. Let $\gamma$ given by (4) be the sequence which is obtained by application of Procedure 3.1 to $\tilde{A}$. By Lemma 3.2, $\gamma$ is a lacunary sequence with respect to $C_{\tilde{A}}$. We complete the proof by using induction on $n$. For $n=1$ the result holds trivially. Suppose the result holds for all matrices in $\mathbb{R}^{k, m}$ with $k \leq n$. We show that it holds for $k=n+1$. We begin by distinguishing the following two cases.

Case 1. $i_{p}=1$.

Application of the condensed form of the Cauchon algorithm to

$$
A[2,3, \ldots, n+1 \mid 1,2, \ldots, m]
$$

results in $\tilde{A}[2,3, \ldots, n+1 \mid 1,2, \ldots, m]$. By the induction hypothesis, $A[2,3, \ldots, n$ $+1 \mid 1,2, \ldots, m]$ has rank $p$ since $\left(\left(i_{p-1}, j_{p-1}\right), \ldots,\left(i_{0}, j_{0}\right)\right)$ is the lacunary sequence obtained from Procedure 3.1 applied to $\tilde{A}[2, \ldots, n+1 \mid 1, \ldots, m]$. Thus $p \leq \operatorname{rank} A \leq p+1$. It follows from Corollary 3.3 that $\operatorname{det} A\left[i_{p}, i_{p-1}, \ldots, i_{0} \mid j_{p}, j_{p-1}, \ldots, j_{0}\right] \neq 0$ since $\gamma$ is a lacunary sequence with respect to $C_{\tilde{A}}$ and $\tilde{a}_{i_{p}, j_{p}} \neq 0$, which implies $\operatorname{rank} A=p+1$ as desired.

Case 2. $i_{p}>1$. By the induction hypothesis $A[2,3, \ldots, n+1 \mid 1,2, \ldots, m]$ has rank $p+1$. So $p+1 \leq \operatorname{rank} A \leq p+2$. Let $\alpha:=\left\{i_{p}, i_{p-1}, \ldots, i_{0}\right\}$, $\beta:=\left\{j_{p}, j_{p-1}, \ldots, j_{0}\right\}$ and define $B=\left(b_{i j}\right), i \in \alpha^{c}, j \in \beta^{c}$, with

$$
b_{i j}:=\frac{\operatorname{det} A[\alpha \cup\{i\} \mid \beta \cup\{j\}]}{\operatorname{det} A[\alpha \mid \beta]} .
$$

Note that by Corollary 3.3 we have $\operatorname{det} A[\alpha \mid \beta] \neq 0$ since $\tilde{a}_{i j} \neq 0$ for all $(i, j) \in\left\{\left(i_{p}, j_{p}\right),\left(i_{p-1}, j_{p-1}\right), \ldots,\left(i_{0}, j_{0}\right)\right\}$ and by the induction hypothesis $b_{i j}=0$ for all $i \in \alpha^{c} \backslash\{1\}$. 
Claim. With $B$ as defined as above we claim that $b_{1 j}=0$ for all $j \in \beta^{c}$. Case 2.1. $1 \leq j<j_{p}$.

In this case it is easy to see that the sequence $\left((1, j),\left(i_{p}, j_{p}\right),\left(i_{p-1}, j_{p-1}\right), \ldots\right.$, $\left.\left(i_{0}, j_{0}\right)\right)$ is a lacunary sequence with respect to $C_{\tilde{A}}$ and by Corollary 3.3 $b_{1 j}=0$.

Case 2.2. $j \in \beta^{c}$ and $j>j_{p}$.

In this case there exists $s \in\{1, \ldots, p\}$ such that $j_{s}<j<j_{s-1}$ provided that $p>0$ (if $p=0$, then $\tilde{A}[1, \ldots, n+1 \mid j]=A[1, \ldots, n+1 \mid j]=0$ since $\tilde{A}$ is Cauchon matrix.) In the following we prove that the column $j$ of $A$ is linearly dependent on columns $j_{0}, j_{1}, \ldots, j_{s-1}$. Since $\tilde{A}$ is a Cauchon matrix and $\gamma$ is the sequence that we obtained by Procedure 3.1 we have $\tilde{a}_{i j^{\prime}}=0$ for all $i<i_{s-1}, j \leq j^{\prime}<j_{s-1}$. For this particular $j$, take $\mu:=\left\{i_{s-1}, \ldots, i_{0}\right\}$, $\nu:=\left\{j_{s-1}, \ldots, j_{0}\right\}$, and define the vector, $G=\left(g_{i 1}\right), i \in \mu^{c}$, where

$$
g_{i 1}:=\frac{\operatorname{det} A[\mu \cup\{i\} \mid \nu \cup\{j\}]}{\operatorname{det} A[\mu \mid \nu]} .
$$

We have, by Corollary $3.3, g_{i 1}=0$ for all $i<i_{s-1}$ since $\left((i, j),\left(i_{s-1}, j_{s-1}\right), \ldots\right.$, $\left.\left(i_{0}, j_{0}\right)\right)$ is a lacunary sequence with respect to $C_{\tilde{A}[1, \ldots, n+1 \mid j, j+1, \ldots, m]}$ and $\tilde{a}_{i j}=0$ . Furthermore, we conclude that $g_{i 1}=0$ for all $i>i_{s-1}$ and $i \in \mu^{c}$, since by the induction hypothesis $\operatorname{rank} A\left[i_{s-1}, \ldots, n+1 \mid j, \ldots, m\right]=s$. Hence we have shown $G=0$. Application of Lemma 2.1 with $\gamma^{\prime}:=\mu^{c}$ and $\delta^{\prime}:=\{j\}$ yields $\operatorname{rank} A\left[1, \ldots, n+1 \mid j, j_{s-1}, \ldots, j_{0}\right]=s$ and since $\operatorname{det} A\left[i_{s-1}, \ldots, i_{0} \mid j_{s-1}, \ldots, j_{0}\right] \neq$ 0 it follows that $\operatorname{rank} A\left[1, \ldots, n+1 \mid j_{s-1}, \ldots, j_{0}\right]=s$. Hence column $j$ depends linearly on columns $j_{s-1}, \ldots j_{0}$. We conclude that $b_{1 j}=0$ for all $j>j_{p}$ and $j \in \beta^{c}$ which completes the proof of the claim. Hence we have proven that $B=0$ and application of Lemma 2.1 with $\gamma:=\alpha, \gamma^{\prime}:=\alpha^{c}, \delta:=\beta$, and $\delta^{\prime}:=\beta^{c}$ yields $\operatorname{rank} A=p+1$.

If one has to check a set of row vectors of a given $n$-by- $m$ matrix $A$ that admits a Cauchon matrix upon application of the Cauchon algorithm for linear independence, then one can run Algorithm 2.4 on the submatrix of $A$ formed by these vectors and apply Theorem 3.4. In the next theorem we consider the case that we have already computed $\tilde{A}$ and want to check a set of consecutive row vectors of $A$ for linear independence.

Theorem 3.5. Let $A \in \mathbb{R}^{n, m}$ be such that $\tilde{A}$ is a Cauchon matrix. Then for $i=1, \ldots, n$ and $0 \leq l \leq n-i$, the rows $i, i+1, \ldots, i+l$ of $A$ are 
linearly independent if and only if application of Procedure 3.1 to $\tilde{A}[i, \ldots, i+$ $l \mid 1, \ldots, m]$ results in a sequence of length $l$.

Proof. Let $\gamma:=\left(\left(i_{l}, j_{l}\right),\left(i_{l-1}, j_{l-1}\right), \ldots,\left(i_{0}, j_{0}\right)\right)$ be the sequence which is obtained by application of Procedure 3.1 to $\tilde{A}[i, \ldots, i+l \mid 1, \ldots, m]$. Then we have $i+f=i_{l-f}$, for $f=0,1, \ldots, l$, and distinguish the following two cases. Case 1. $j_{0}=m$. Then $\gamma$ is a lacunary sequence with respect to $C_{\tilde{A}}$. Hence it follows by Corollary 3.3 that $\operatorname{det} A\left[i_{l}, \ldots, i_{0} \mid j_{l}, \ldots, j_{0}\right] \neq 0$. Since the submatrix $A\left[i_{l}, \ldots, i_{0} \mid j_{l}, \ldots, j_{0}\right]$ is contained in the rows indexed by $i, \ldots, i+l$ we conclude that these rows are linearly independent.

Case 2. $j_{0}<m$. Then it follows that $\tilde{a}_{i_{0}, j}=0$ for all $j=j_{0}+1, \ldots, m$. Since $\tilde{a}_{i_{0}, j_{0}} \neq 0$ and $\tilde{A}$ is a Cauchon matrix we have that $\tilde{A}\left[1, \ldots, i_{0} \mid j_{0}+1, \ldots, m\right]=$ 0 and hence $A\left[1, \ldots, i_{0} \mid j_{0}+1, \ldots, m\right]=0$. By constructing a lacunary sequence starting from $\left(i_{0}, j_{0}\right)$ and appending this sequence to $\gamma$ we obtain a lacunary sequence with respect to $C_{\tilde{A}}$, say $\left(\left(i_{l}, j_{l}\right), \ldots,\left(i_{0}, j_{0}\right),\left(i_{1}^{\prime}, j_{1}^{\prime}\right)\right.$, $\left.\ldots,\left(i_{p}^{\prime}, j_{p}^{\prime}\right)\right)$. By Corollary $3.3, \operatorname{det} A\left[i_{l}, \ldots, i_{0}, i_{1}^{\prime}, \ldots, i_{p}^{\prime} \mid j_{l}, \ldots, j_{0}, j_{1}^{\prime}, \ldots, j_{p}^{\prime}\right] \neq$ 0 and the zero-nonzero pattern of $A$ implies

$$
\begin{aligned}
\operatorname{det} A\left[i_{l}, \ldots, i_{0}, i_{1}^{\prime}, \ldots, i_{p}^{\prime} \mid j_{l}, \ldots, j_{0}, j_{1}^{\prime}, \ldots, j_{p}^{\prime}\right] & = \\
\operatorname{det} A\left[i_{l}, \ldots, i_{0} \mid j_{l}, \ldots, j_{0}\right] \cdot & \operatorname{det} A\left[i_{1}^{\prime}, \ldots, i_{p}^{\prime} \mid j_{1}^{\prime}, \ldots, j_{p}^{\prime}\right],
\end{aligned}
$$

whence $\operatorname{det} A\left[i_{l}, \ldots, i_{0} \mid j_{l}, \ldots, j_{0}\right] \neq 0$. Therefore, the rows indexed by $i, i+$ $1, \ldots, i+l$ of $A$ are linearly independent.

Conversely, suppose that the rows $i, i+1, \ldots, i+l$ of $A$ are linearly independent and the application of Procedure 3.1 to $\tilde{A}[i, \ldots, i+l \mid 1, \ldots, m]$ results in a sequence of length $t$ less than $l$, say $\left(\left(i_{t}, j_{t}\right), \ldots,\left(i_{0}, j_{0}\right)\right)$, provided that $l>0$ (if $l=0$ then $\tilde{A}[i \mid 1, \ldots, m]$ is a zero row, whence $A[i \mid 1, \ldots, m+1]$ is a zero row, too, a contradiction to our assumption). Without loss of generality, suppose that $j_{0}=m$; otherwise we proceed as in Case 2 above.

Let $\kappa:=\min \left\{s: i_{s}>i_{s+1}+1\right\}, \alpha:=\left\{i_{\kappa}, i_{\kappa-1}, \ldots, i_{0}\right\}, \beta:=\left\{j_{\kappa}, j_{\kappa-1}, \ldots, j_{0}\right\}$, and let $B=\left(b_{1 j}\right), j \in \beta^{c}$, where

$$
b_{1 j}:=\frac{\operatorname{det} A\left[\alpha \cup\left\{i_{\kappa}-1\right\} \mid \beta \cup\{j\}\right]}{\operatorname{det} A[\alpha \mid \beta]} .
$$

By Corollary 3.3, $b_{1 j}=0$ for all $j<j_{\kappa}$ since $\tilde{a}_{i_{\kappa}-1, j}=0$ and $\left(\left(i_{\kappa}-1, j\right)\right.$, $\left.\left(i_{\kappa}, j_{\kappa}\right), \ldots,\left(i_{0}, j_{0}\right)\right)$ is a lacunary sequence with respect to $C_{\tilde{A}[1, \ldots, n \mid j, \ldots, m]}$.

Claim. $b_{1 j}=0$ for all $j>j_{\kappa}$ and $j \in \beta^{c}$.

Let $1 \leq h \leq \kappa$ be such that $j_{h}<j<j_{h-1}$. We want to show that the 
column $A[1, \ldots, i+l \mid j]$ depends linearly on the columns $A\left[1, \ldots, i+l \mid j_{s}\right]$, $s=0, \ldots, h-1$. For this fixed $j$, let $G=\left(g_{q 1}\right), q=1,2, \ldots, i_{h-1}-1$,

$$
g_{q 1}:=\frac{\operatorname{det} A[\sigma \cup\{q\} \mid \tau \cup\{j\}]}{\operatorname{det} A[\sigma \mid \tau]}
$$

where $\sigma:=\left\{i_{h-1}, \ldots, i_{0}\right\}$ and $\tau:=\left\{j_{h-1}, \ldots, j_{0}\right\}$.

It is easy to see that $\left((q, j),\left(i_{h-1}, j_{h-1}\right), \ldots,\left(i_{0}, j_{0}\right)\right)$ is a lacunary sequence with respect to $C_{\tilde{A}[1, \ldots, n \mid j, j+1, \ldots, m]}$ and $\tilde{a}_{q j}=0$ for all $q=1, \ldots, i_{h-1}-1$. Hence it follows by Corollary 3.3 that $G=0$. Using Lemma 2.1 we have $\operatorname{rank} A[1, \ldots, i+l \mid \tau \cup\{j\}]=h$. Since $\operatorname{det} A[\sigma \mid \tau] \neq 0$ we may conclude that the column $A[1, \ldots, i+l \mid j]$ depends linearly on $A\left[1, \ldots, i+l \mid j_{s}\right], s=0, \ldots, h-1$. Therefore, $b_{1 j}=0$ for all $j>j_{\kappa}$ and $j \in \beta^{c}$. We have shown that $B=0$, which implies by application of Lemma 2.1 with $\gamma:=\alpha, \gamma^{\prime}=\left\{i_{\kappa}-1\right\}$, $\delta:=\beta$, and $\delta^{\prime}:=\beta^{c}$, that $\operatorname{rank} A\left[i_{\kappa}-1, i_{\kappa} \ldots, i_{0} \mid 1, \ldots, m\right]=\kappa+1$. Thus row $i_{\kappa}-1$ depends linearly on rows $i_{\kappa}, \ldots, i_{0}$ of $A$, which is a contradiction.

The following example shows that the statement of Theorem 3.5 is not true if we allow the rows of $A$ to be non-consecutive.

Example 3.6. Consider the matrix

$$
A=\left[\begin{array}{llll}
2 & 2 & 2 & 1 \\
2 & 2 & 2 & 1 \\
1 & 1 & 1 & 1
\end{array}\right]
$$

Then we obtain by Algorithm 2.4

$$
\tilde{A}=\left[\begin{array}{llll}
0 & 0 & 0 & 1 \\
0 & 0 & 1 & 1 \\
1 & 1 & 1 & 1
\end{array}\right] \text {. }
$$

If Theorem 3.5 would be valid also in the case that the rows to be checked for linear independence are non-consecutive, e.g., the first and the third row of A, we could apply Procedure 3.1 to $\tilde{A}[1,3 \mid 1,2,3,4]$ which would result in a sequence $\gamma$ containing only the pair $(3,4)$. By Theorem 3.5 it would follow that both rows are linearly dependent, whereas the application of Procedure 3.1 to $A[1, \widetilde{3 \mid 1,2}, 3,4]$ which is identical with the last two rows of $\tilde{A}$ gives the correct result. 
Corollary 3.7. Let $A \in \mathbb{R}^{n, m}$ be such that $\tilde{A}$ is a Cauchon matrix. Then columns $j, j+1, \ldots, j+l$ of $A$ are linearly independent if and only if application of Procedure 3.1 to $\tilde{A}[1,2, \ldots, n \mid j, j+1, \ldots, j+l]$ results in a sequence of length $l$.

Corollary 3.8. Let $A \in \mathbb{R}^{n, m}$ be such that $\tilde{A}$ is a Cauchon matrix and $n \leq$ $m$. Then $\operatorname{rank} A=n$ if and only if application of Procedure 3.1 to $\tilde{A}$ results in a sequence of length $n-1$.

The following corollary was already proven in [2, Proposition 2.8] in the special case that $A$ is totally nonnegative.

Corollary 3.9. Let $A \in \mathbb{R}^{n, n}$ be such that $\tilde{A}$ is a Cauchon matrix. Then $A$ is nonsingular if and only if $\tilde{a}_{i i} \neq 0$ for all $i=1,2, \ldots, n$.

Proof. Let $A$ be nonsingular. Then the rows of $A$ are linearly independent. By Theorem 3.5 the application of Procedure 3.1 to $\tilde{A}$ results in a sequence of length $n-1$ which forces the squares on the main diagonal of $C_{\tilde{A}}$ to be white, otherwise the length of the sequence will be less than $n-1$.

Conversely, suppose $\tilde{a}_{i i} \neq 0, i=1, \ldots, n$. Then applying Procedure 3.1 to $\tilde{A}$ results in the sequence $((1,1),(2,2), \ldots,(n, n))$, which has length $n-$ 1. Therefore, it follows by Theorem 3.4 , that $\operatorname{rank} A=n$, and thus $A$ is nonsingular.

\section{Descending Rank Conditions}

Investigating when a class of matrices (such as positive definite or totally positive matrices) admits a particular type of factorization is an important study. Often many intrinsic properties about a particular class of matrices can be deduced from certain factorization results. A factorization which is especially suited for, e.g., the class of the totally nonnegative matrices, is elementary bidiagonal factorization, see, e.g., [7, Chapter 2]. We are interested here in a particular order of the factors which corresponds to elimination of entries in the following order

$$
\begin{aligned}
& (n, 1), \ldots,(2,1),(n, 2), \ldots,(3,2), \ldots,(n, n-1), \\
& (1, n), \ldots,(1,2),(2, n), \ldots,(2,3), \ldots,(n-1, n) .
\end{aligned}
$$


In this section we connect the notions of Cauchon diagrams, bidiagonal factorizations, and a set of so-called rank conditions. The existence of a bidiagonal factorization for a given matrix has been linked to certain types of rank conditions associated with the ranks of sets of submatrices, and we make use of this connection here by incorporating them both with Cauchon matrices (or diagrams) and the condensed Cauchon algorithm. The definitions in this section can be found in [10].

Definition 4.1. Let $A \in \mathbb{R}^{n, n}$. Then we say that $A$ has a successively ordered elementary factorization ( $S E B$ ) if $A$ can be written as

$$
A=\left(\prod_{k=1}^{n-1} \prod_{j=n}^{k+1} L_{j}\left(l_{j k}\right)\right) D\left(\prod_{k=n-1}^{1} \prod_{j=k+1}^{n} U_{j}\left(u_{k j}\right)\right)
$$

where $L_{i}(s)=I+s E_{i, i-1}, U_{j}(t)=I+t E_{j-1, j}, 2 \leq i, j \leq n$, and $D$ is a diagonal matrix.

For example, if a 4-by-4 matrix $A$ has an $S E B$ factorization, then it is of the form

$$
\begin{aligned}
A= & L_{4}\left(l_{41}\right) L_{3}\left(l_{31}\right) L_{2}\left(l_{21}\right) L_{4}\left(l_{42}\right) L_{3}\left(l_{32}\right) L_{4}\left(l_{43}\right) D \\
& \times U_{4}\left(u_{34}\right) U_{3}\left(u_{23}\right) U_{4}\left(u_{24}\right) U_{2}\left(u_{12}\right) U_{3}\left(u_{13}\right) U_{4}\left(u_{14}\right) .
\end{aligned}
$$

In the event that not all possible $E B$ factors are included in (7), the $S E B$ factorization of a given matrix (when it exists) may not be unique. Thus we narrow the study of $S E B$ factorizations to achieve existence and uniqueness. We call an $S E B$ factorization (7) an elimination $S E B(E S E B)$ if it results from the reduction of an $n$-by- $n$ matrix $A$ to the diagonal matrix $D$ by eliminating nonzero entries via elementary bidiagonal row/column operations performed in the order (6). Note that the subscripts on $l_{j k}$ and $u_{k j}$ correspond to the entry being eliminated. If $l_{j k}$ or $u_{j k}$ is 0 in any factor, then that factor is not included in the $E S E B$ factorization. It was shown in [10, Theorem 9] that if an $E S E B$ factorization exists, then it is unique.

Example 4.2. [10, Example 12] Let $A$ be the 5-by-5 unit lower triangular matrix

$$
A=\left[\begin{array}{lllll}
1 & 0 & 0 & 0 & 0 \\
2 & 1 & 0 & 0 & 0 \\
1 & 1 & 1 & 0 & 0 \\
1 & 1 & 2 & 1 & 0 \\
1 & 1 & 4 & 4 & 1
\end{array}\right]
$$


Then $A$ admits the ESEB factorization

$$
A=L_{5}(1) L_{4}(1) L_{3}\left(\frac{1}{2}\right) L_{2}(2) L_{3}\left(\frac{1}{2}\right) L_{5}(2) L_{4}(1) L_{5}(1) .
$$

Definition 4.3. Let $A \in \mathbb{R}^{n, n}$. Then $A$ satisfies the column descending rank condition if for all $l$ with $1 \leq l \leq n-1$, for all $z$ with $0 \leq z \leq l-1$, and for all $p$ satisfying $l-z \leq p \leq n-z-1$,

$$
\operatorname{rank} A[p+1, \ldots, p+z+1 \mid 1, \ldots, l] \leq \operatorname{rank} A[p, \ldots, p+z \mid 1, \ldots, l] .
$$

Similarly, A satisfies the row descending rank condition if with the indices as above

$$
\operatorname{rank} A[1, \ldots, l \mid p+1, \ldots, p+z+1] \leq \operatorname{rank} A[1, \ldots, l \mid p, \ldots, p+z] .
$$

A satisfies the descending rank conditions if $A$ satisfies both the row and column descending rank conditions.

Example 4.2 (continued). It is easy to see that the matrix A given in (8) satisfies the column descending rank condition. In fact, inequality (10) is fulfilled for all feasible values of $l, z$, and $p$ with equality, with the exception of the cases $l=2, z=1, p=2$ and $l=3, z=2, p=2$ in which strict inequality holds.

Using the descending rank conditions in connection with the condensed form of the Cauchon algorithm, we have the following results related to Cauchon matrices.

Theorem 4.4. Let $A \in \mathbb{R}^{n, n}$ and $B:=A^{\#}$. If $A$ satisfies the descending rank conditions, then the following statements hold:

(i) If $\tilde{b}_{i j}=0$ for some $i \geq j$, then $\tilde{b}_{i t}=0$ for all $t<j$;

(ii) if $\tilde{b}_{i j}=0$ for some $i \leq j$, then $\tilde{b}_{t j}=0$ for all $t<i$;

(iii) $\tilde{B}$ is a Cauchon matrix.

Proof. Statement (iii) follows as a result of (i) and (ii). We only prove (i) since (ii) follows analogously.

The proof uses decreasing induction with respect to the lexicographical order. If $i=n$, then (i) holds trivially. Assume that (i) holds for all $(i, j)$ 
with $\left(i_{0}, j_{0}\right) \leq(i, j)$ and $\left(i_{0}, j_{0}\right) \neq(i, j)$ and $\tilde{b}_{i_{0}, j_{0}}=0$, where $i_{0} \geq j_{0}$. Suppose on the contrary that there exists $j^{\prime}<j_{0}$ such that $\tilde{b}_{i_{0}, j^{\prime}} \neq 0$. Without loss of generality we assume that $j^{\prime}=j_{0}-1$. If $\tilde{B}\left[i_{0}, \ldots, n \mid j_{0}\right]=0$, then $B\left[i_{0}, \ldots, n \mid j_{0}\right]=0$. Hence by the row descending rank condition we have $B\left[i_{0}, \ldots, n \mid 1, \ldots, j_{0}\right]=0$, which in turn implies that $\tilde{B}\left[i_{0}, \ldots, n \mid 1, \ldots, j_{0}\right]=$ 0 . However, this contradicts our hypothesis that $\tilde{b}_{i_{0}, j_{0}-1} \neq 0$. Hence assume that $\tilde{B}\left[i_{0}, \ldots, n \mid j_{0}\right] \neq 0$. Set $q_{0}:=i_{0}$ and for $h=1, \ldots, f$ define $q_{h}$, sequentially, by

$$
q_{g}=\min \left\{t \mid t>q_{g-1}, \quad \tilde{b}_{t, j_{0}+g-1} \neq 0\right\} .
$$

It is clear that $\left(\left(q_{0}, j_{0}-1\right),\left(q_{1}, j_{0}\right), \ldots,\left(q_{f}, j_{0}+f-1\right)\right)$ and $\left(\left(q_{1}, j_{0}+1\right),\left(q_{2}, j_{0}+2\right), \ldots,\left(q_{f}, j_{0}+f\right)\right)$ are sequences which can be obtained by application of Procedure 3.1 to the columns $j_{0}-1, j_{0}, \ldots, j_{0}+f-1$ and $j_{0}, j_{0}+1, \ldots, j_{0}+f$ of $\tilde{B}\left[i_{0}, \ldots, n \mid 1, \ldots, n\right]$, respectively. By Corollary 3.7, the columns $B\left[i_{0}, \ldots, n \mid e\right], e=j_{0}-1, j_{0}, \ldots, j_{0}+f-1$ are linearly independent and hence $\operatorname{rank} B\left[i_{0}, \ldots, n \mid j_{0}-1, \ldots, j_{0}+f-1\right]=f+1$, while the columns $B\left[i_{0}, \ldots, n \mid e\right], e=j_{0}, j_{0}+1, \ldots, j_{0}+f$, are linearly dependent. Hence $\operatorname{rank} B\left[i_{0}, \ldots, n \mid j_{0}, \ldots, j_{0}+f\right] \leq f$, which contradicts the assumed row descending rank condition.

Example 4.2 (continued). For the matrix A given in (8) the matrix $B=A^{\#}$ is

$$
B=\left[\begin{array}{lllll}
1 & 4 & 4 & 1 & 1 \\
0 & 1 & 2 & 1 & 1 \\
0 & 0 & 1 & 1 & 1 \\
0 & 0 & 0 & 1 & 2 \\
0 & 0 & 0 & 0 & 1
\end{array}\right]
$$

Obviously, the matrix

$$
\tilde{B}=\left[\begin{array}{lllll}
1 & 1 & 2 & 0 & 0 \\
0 & 1 & 1 & 0 & 1 \\
0 & 0 & 1 & \frac{1}{2} & 1 \\
0 & 0 & 0 & 1 & 2 \\
0 & 0 & 0 & 0 & 1
\end{array}\right]
$$

satisfies (i)-(iii) in Theorem 4.4. 
Remark 4.5. Let $B \in \mathbb{R}^{n, n}, B \neq 0$, such that (i) and (ii) in Theorem 4.4 hold. Then

$$
\operatorname{rank} B[i, i+1, \ldots, n \mid j, j+1, \ldots, j+w]=l+1,
$$

where $0 \leq w \leq n-j$ and $l$ is the length of the sequence which is obtained by application of Procedure 3.1 to $\tilde{B}[i, i+1, \ldots, n \mid j, j+1, \ldots, j+w]$. An analogous statement holds for $\operatorname{rank} B[i, i+1, \ldots, i+w \mid j, j+1, \ldots, n]$, where $0 \leq w \leq n-i$.

Theorem 4.6. Let $A \in \mathbb{R}^{n, n}$ and $B:=A^{\#}$. If $B$ satisfies (i) and (ii) in Theorem 4.4, then $A$ satisfies the descending rank conditions.

Proof. We show only that $A$ satisfies the row descending rank condition since the column descending rank condition will follow by applying a similar argument. Let $1 \leq l \leq n-1,0 \leq z \leq l-1$, and $l-z \leq p \leq n-z-1$. We show that

$$
\operatorname{rank} A[1, \ldots, l \mid p+1, \ldots, p+z+1] \leq \operatorname{rank} A[1, \ldots, l \mid p, \ldots, p+z],
$$

which is equivalent to

$$
\begin{aligned}
\operatorname{rank} B[n-l+1, \ldots, n \mid n-p-z, \ldots, n-p] \leq \\
\quad \operatorname{rank} B[n-l+1, \ldots, n \mid n-p-z+1, \ldots, n-p+1] .
\end{aligned}
$$

From condition (i) of Theorem 4.4 the length of the sequence obtained by application of Procedure 3.1 to $\tilde{B}[n-l+1, \ldots, n \mid n-p-z+1, \ldots, n-p+1]$ is greater than or equal to the length of the one obtained by application of Procedure 3.1 to $\tilde{B}[n-l+1, \ldots, n \mid n-p-z, \ldots, n-p]$. Therefore, (13) holds by employing Remark 4.5 .

Theorem 4.7. Let $A \in \mathbb{R}^{n, n}$ and $B:=A^{\#}$. If $G:=B^{T}$ satisfies (i) and (ii) in Theorem 4.4, then $A$ has an ESEB factorization (7) in which $l_{j k}$ and $u_{k j}, k=1, \ldots, n-1, j=k+1, \ldots, n$, and $d_{i i}, i=1, \ldots, n$ are given by

(a) $l_{n 1}=\frac{\tilde{g}_{n 1}}{\tilde{g}_{n 2}}, l_{n-1,1}=\frac{\tilde{g}_{n 2}}{\tilde{g}_{n 3}}, \ldots, l_{21}=\frac{\tilde{g}_{n, n-1}}{\tilde{g}_{n n}}$,

$$
\begin{aligned}
& l_{n 2}=\frac{\tilde{g}_{n-1,1}}{\tilde{g}_{n-1,2}}, l_{n-1,2}=\frac{\tilde{g}_{n-1,2}}{\tilde{g}_{n-1,3}}, \ldots, l_{3,2}=\frac{\tilde{g}_{n-1, n-2}}{\tilde{g}_{n-1, n-1}}, \ldots, \\
& l_{n, n}=\frac{\tilde{g}_{21}}{\tilde{g}_{22}}
\end{aligned}
$$

(b) $d_{i i}=\tilde{g}_{n-i, n-i}, i=1, \ldots, n$; 


$$
\begin{array}{ll}
\text { (c) } & u_{n-1, n}=\frac{\tilde{g}_{12}}{\tilde{g}_{22}}, \\
& u_{n-2, n-1}=\frac{\tilde{g}_{13}}{\tilde{g}_{23}}, u_{n-2, n}=\frac{\tilde{g}_{23}}{\tilde{g}_{33}}, \ldots, \\
& u_{12}=\frac{\tilde{g}_{1, n}}{\tilde{g}_{2, n}}, u_{13}=\frac{\tilde{g}_{2, n}}{\tilde{g}_{3, n}}, \ldots, u_{1 n}=\frac{\tilde{g}_{n-1, n}}{\tilde{g}_{n, n}},
\end{array}
$$

with the convention $\frac{0}{0}:=0$.

Proof. Since $G$ satisfies (i) and (ii) in Theorem 4.4, $\tilde{G}$ is a Cauchon matrix with all entries in the same row to the left (in the lower part, including the main diagonal) or all entries in the same column above (in the upper part, including the main diagonal) of a zero entry in $\tilde{G}$ vanish, too. We replace such zero entries from the right to the left and from the bottom to the top by increasing integral powers of a small positive number $\epsilon$. Call the resulting matrix $\tilde{G}_{\epsilon}$. We apply the Restoration algorithm (see [9] or [1]), which is the inverse procedure to the Cauchon algorithm, to $\tilde{G}_{\epsilon}$ and obtain the matrix $G_{\epsilon}$. Since $\tilde{G}_{\epsilon}$ tends to $\tilde{G}$ as $\epsilon$ tends to 0 , it is easy to see that $G_{\epsilon}$ tends to $G$. Hence we can approximate the given matrix $G$ by the matrix $G_{\epsilon}$ as closely as desired such that

$$
\operatorname{det} G_{\epsilon}[i, i+1, \ldots, i+w \mid j, j+1, \ldots, j+w] \neq 0
$$

where $w:=\min \{n-i, n-j\}$. This implies for the matrix $A_{\epsilon}:=\left(G_{\epsilon}^{T}\right)^{\#}$ that

$$
\operatorname{det} A_{\epsilon}[n-j-w+1, \ldots, n-j+1 \mid n-i-w+1, \ldots, n-i+1] \neq 0 .
$$

Hence $A_{\epsilon}$ has a $E S E B$ factorization which can be obtained from the elements of $\tilde{G}_{\epsilon}$ as in the case that $A$ is nonsingular and totally nonnegative (see [1, p.52], [3, Section 4], although the analysis still applies in this case as well). Hence as $\epsilon$ tends to zero, we obtain the representation of $l_{j k}$ and $u_{k j}, k=$ $1, \ldots, n-1, j=k+1, \ldots, n, d_{i i}, i=1, \ldots, n$, of the $E S E B$ factorization as given in the statement of the theorem.

In the final theorem we put together the results of Theorems 4.4 and 4.6. The equivalence between (b) and (c) can be found in [10, Theorem 9].

Theorem 4.8. Let $A \in \mathbb{R}^{n, n}$ and $B:=A^{\#}$. Then the following statements are equivalent:

(a) B satisfies (i) and (ii) in Theorem 4.4. 
(b) A satisfies the descending rank conditions.

(c) A has an ESEB factorization.

A very interesting by-product of this characterization (see Theorem 4.8) is the complexity saving associated with determining if a matrix satisfies the

row and/or column rank descending conditions. In particular, the complexity is reduced from calculating the ranks of $n(n+1)^{2}-\frac{n(n+1)(2 n+1)}{3}-n$ submatrices to at most $(n-1)^{2}(n+1)$ arithmetic operations which are required by the condensed form of the Cauchon Algorithm [1, p.52]. In addition, our work in this section highlights the utility of the condensed form of the Cauchon algorithm and the study of Cauchon diagrams (or matrices), beyond, in particular, the class of totally nonnegative matrices [9] and totally nonpositive matrices [4].

\section{Acknowledgment}

The first author's work leading to this publication was supported partially by the German Academic Exchange Service (DAAD) with funding from the German Federal Ministry of Education and Research (BMBF) and the People Programme (Marie Curie Actions) of the European Union's Seventh Framework Programme (FP7/2007-2013) under REA grant agreement no. 605728 (P.R.I.M.E. - Postdoctoral Researchers International Mobility Experience). Fallat's research supported in part by an NSERC Discovery Research Grant, Application No.: RGPIN-2014-06036.

\section{References}

[1] M. Adm, Perturbation and Intervals of Totally Nonnegative Matrices and Related Properties of Sign Regular Matrices, Dissertation, University of Konstanz, Konstanz, Germany, 2016.

[2] M. Adm, J. Garloff, Intervals of totally nonnegative matrices, Linear Algebra Appl. 439 (2013) 3796-3806.

[3] M. Adm, J. Garloff, Improved tests and characterizations of totally nonnegative matrices, Electron. J. Linear Algebra 27 (2014) 588-610. 
[4] M. Adm, J. Garloff, Intervals of special sign regular matrices, Linear Multilinear Algebra 64 (2016) 1424-1444.

[5] C. de Boor, A. Pinkus, The approximation of a totally positive band matrix by a strictly banded totally positive one, Linear Algebra Appl. 42 (1982) 81-98.

[6] G. Cauchon, Spectre premier de $O_{q}\left(M_{n}(k)\right)$ image canonique et séparation normale, J. Algebra 260 (2003) 519-569.

[7] S.M. Fallat, C.R. Johnson, Totally Nonnegative Matrices, Princeton Ser. Appl. Math., Princeton University Press, Princeton and Oxford, 2011.

[8] M. Fiedler, T.L. Markham, Consecutive-column and -row properties of matrices and the Loewner-Neville factorization, Linear Algebra Appl. 266 (1997) 243-259.

[9] K.R. Goodearl, S. Launois, T.H. Lenagan, Totally nonnegative cells and matrix Poisson varieties, Adv. Math. 226 (2011) 779-826.

[10] C.R. Johnson, D.D. Olesky, P. Van Den Driessche, Successively ordered elementary bidiagonal factorization, SIAM J. Matrix Anal. Appl. 22 (2001) 1079-1088.

[11] S. Launois, T.H. Lenagan, Efficient recognition of totally nonnegative matrix cells, Found. Comput. Math. 14 (2014) 371-387.

[12] A. Pinkus, Totally Positive Matrices, Cambridge Tracts in Math., vol. 181, Cambridge Univ. Press, Cambridge, UK, 2010.

[13] A. Postnikov, Total positivity, Grassmannians, and networks, arXiv:math/0609764v1 [math.CO], 27 Sep 2006. 